\title{
HIGHLIGHTS
}

VIRAL PATHOGENESIS

\section{Reovirus double whammy}

Many viruses count the ability to manipulate apoptosis - programmed cell death - in their antihost arsenal. New work published in the Journal of Virology specifically examines alterations in apoptosis signalling - at the transcriptional level — induced by reovirus infection. The key distinguishing feature of this study is comparison between cellular responses to two strains of reovirus differing only in their ability to cause apoptosis.

Apoptosis is not only induced by viruses to kill host cells, it is an essential part of life for any multicellular organism. Apoptosis mechanisms are conserved from worm to man. Consequently, apoptosis is very well characterised. Significantly, DeBiasi et al. showed - using microarrays and confirmatory RT-PCR - that coordinated groups of genes involved in apoptosis signalling pathways were specifically activated or repressed only by the strain of reovirus that causes apoptosis. So, cellular gene responses specifically related to virus-induced apoptosis, as opposed to general antiviral responses, were delimited.

One of the most exciting insights into reovirus pathogenesis was specific repression of DNA damage repair genes - the first time that this has been linked to viral infection and apoptosis. DNA damage is one of the main stimuli for apoptosis. If DNA is damaged it must be repaired, but if DNA repair doesn't work, its makes sense to dispose of the damaged cell by inducing cell death.
Reovirus deals the host cell a double blow by activating apoptotic pathways and inhibiting DNA repair. Both mechanisms ultimately lead to cell death.

\section{(Q) References and links}

ORIGINAL RESEARCH PAPER DeBiasi, R.L. et

al. Reovirus-induced alteration in expression of

apoptosis and DNA repair genes with potential

roles in viral pathogenesis. J. Virol. 77, 8934-8947

(2003).

WEB SITES

Ken Tyler's laboratory:

http://www.uchsc.edu/sm/neuro/tylerlab/

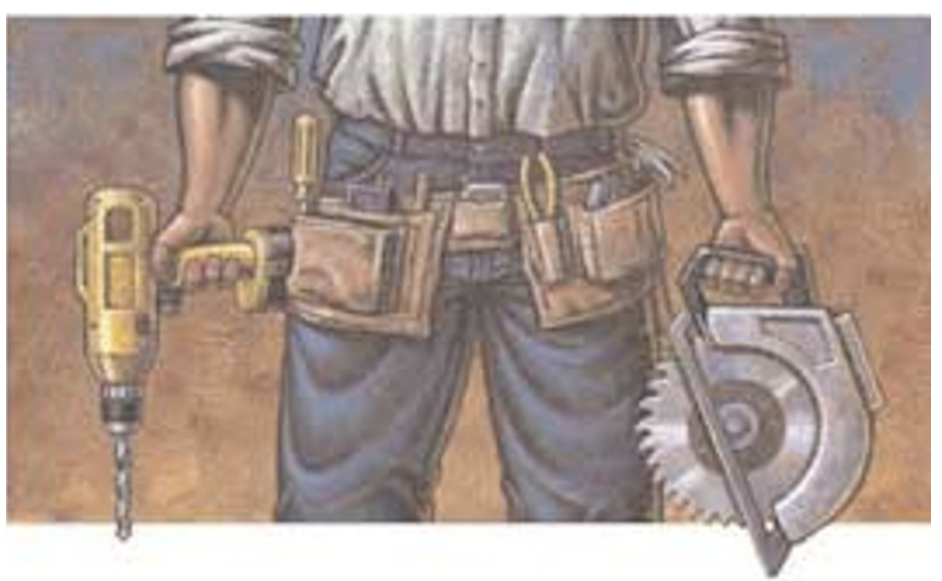

